\title{
BMJ Open The impact of meeting locations for men having sex with men on the risk for bacterial sexually transmitted infections: analyses from a cross- sectional online survey
}

\author{
U Marcus, ${ }^{1} \mathrm{M}$ an der Heiden, ${ }^{1} \mathrm{M}$ Gassowski, ${ }^{1} \mathrm{M}$ Kruspe, ${ }^{2} \mathrm{~J}$ Drewes ${ }^{2}$
}

To cite: Marcus $U$, an der Heiden M, Gassowski M, et al. The impact of meeting locations for men having sex with men on the risk for bacterial sexually transmitted infections: analyses from a cross-sectional online survey. BMJ Open 2015;5:e009107. doi:10.1136/bmjopen-2015009107

- Prepublication history and additional material is available. To view please visit the journal (http://dx.doi.org/ 10.1136/bmjopen-2015009107).

Received 16 June 2015 Revised 25 September 2015 Accepted 14 October 2015

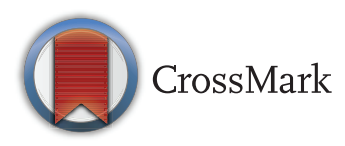

\footnotetext{
${ }^{1}$ Department of Infectious Disease Epidemiology, Robert Koch-Institut, Berlin, Germany

${ }^{2}$ Department of Public Health, Free University, Berlin, Germany
}

Correspondence to Dr U Marcus;

MarcusU@rki.de

\section{ABSTRACT}

Objectives: Opportunities for men having sex with men (MSM) to meet each other have very much improved by new communication technologies. Meeting venue-based characteristics can impact how many partners are met and how much sexual risk is taken. We analysed the association between physical and virtual venues and the risk for bacterial sexually transmitted infections (bSTIs) among participants in an MSM online survey.

Methods: Data were collected during 2013/2014 with a survey targeting MSM living in Germany. The impact of the meeting place with the last non-steady anal sex partner on diagnosis with a bSTI in the previous year was analysed using bivariate and multivariate regression analysis, taking into account self-reported HIV status, serostatus communication, condom use, partner number, age and city size.

Results: The study sample consisted of 8878 respondents (7799 not diagnosed with HIV; 1079 diagnosed with HIV). Meeting partners online was most common (62\% HIV-/51\% HIV+), followed by sex venues (11\% HIV-/25\% HIV+); other venues were each reported by $2-6 \%$ of the respondents. Venue-dependent proportions reporting bSTIs in the recent year were 2-4 folds higher among men diagnosed with HIV. In multivariate analysis, HIV status was the strongest predictor for bSTIs (OR=5.0; $95 \% \mathrm{Cl} 2.8$ to 8.7). Compared with meeting partners online, sex (OR 1.6; $95 \% \mathrm{Cl} 1.0$ to 2.5 ) and social venues (OR 1.9; $95 \% \mathrm{Cl}$ 1.4 to 2.6) were associated with increased bSTI risk for men not diagnosed with HIV, but the risk when meeting partners by smartphone apps was only of borderline significance (OR 1.5; 95\% Cl 0.9 to 2.3). For men diagnosed with HIV, bSTI risk increased for sex venues (OR 1.5; 95\% Cl 1.1 to 2.1), and was lower for non-gay/ other venues (OR $0.2 ; 95 \% \mathrm{Cl} 0.1$ to 0.5 ).

Conclusions: Venues are connected to socialbehavioural facets of corresponding sexual encounters, and may be important arenas for differential HIV and STI education, treatment and prevention.

\section{INTRODUCTION}

In all societies, men having sex with men (MSM) represent a minority of the population. Compared with non-sexual-minority

\section{Strengths and limitations of this study}

- Outbreaks and increasing numbers of diagnoses of sexually transmitted infections (STI) among men having sex with men are often attributed to new tools for partner finding. Smartphone applications helping to localize and communicate with potential partners are hypothesised to contribute to this because they may help to increase partner numbers.

- We intend to test this hypothesis by analysing data from a large online survey. Our data cover a broad range of physical and virtual meeting venues and our sample is not restricted to large cities.

- Large differences regarding STI diagnosis rates between men diagnosed and not diagnosed with HIV are partly explained by different access to routine STI screening: while for men diagnosed with HIV in Germany STI testing can be reimbursed as part of regular HIV treatment monitoring, considerable reimbursement barriers for STI screening for men not diagnosed with HIV exist. It is most likely that by using self-reported diagnosis rates, a high proportion of undiagnosed asymptomatic bacterial STIS (bSTIs) among MSM not diagnosed with HIV is missed.

- The online survey was not adapted for smartphones; thus, smartphone users were most likely under-represented in the study sample and attrition of survey participants was high, possibly introducing self-selection biases.

- The reference group for our comparisons is men having sex with men (MSM) meeting their last non-steady anal sex partner online. While this was the most common meeting venue in our online sample, this venue did not exist before 2000 . When comparing STI diagnosis rates among MSM during the 1990s and current diagnosis rates, the possible impact of new communication technologies on sexual networks needs to be considered.

- When analysing the associations between bSTI diagnosis and behaviours during the last episode of anal intercourse with a non-steady partner, we assume these behaviours are representative of the period of STI acquisition on a population level and neglect that STI could also have been transmitted on another occasion and from a steady partner. 
individuals, MSM have limited opportunities to meet other (recognisable) MSM. In the past two decades, these opportunities have very much improved by new communication technologies (internet; mobile internet access devices, aka smartphones) becoming available that were adapted quickly by MSM to seek sexual partners.

Several authors have previously looked into the association between the study participant recruitment place and sex partner meeting place with sexual risk behaviour, primarily with condom use for anal sex, HIV serostatus disclosure and personal responsibility beliefs. Common findings were that MSM frequenting different venues often differ with regard to demographic characteristics, HIV and syphilis infection rates, and risky sexual behaviours. ${ }^{1}$ For example, men meeting new partners in gay bars/clubs are usually younger and more likely to be single than men visiting saunas or men meeting new partners online. ${ }^{2-4}$ Conversations around condom use and HIV are often difficult in gay venues, and more feasible and convenient using online media. ${ }^{5}$ HIV status disclosure is lowest among men who meet their partner in a park, outdoors or in another public place and highest among men who meet their partner online. ${ }^{6}$ A consequence may be less condom use with partners met online due to a higher level of serostatus disclosure. Venue-based characteristics can impact how MSM negotiate sex and HIV-associated risk behaviour. However, in a previous multivariate model of men reporting anal sex during their last encounter, the venue where the partner was met was not significantly associated with unprotected anal intercourse (UAI)..$^{78}$

There has been less research into the association of physical and virtual venues and risk for bacterial sexually transmitted infections (bSTIs), and not much has been published on these issues among European MSM. A recent analysis of factors associated with STI and HIV diagnosis among clients of a German community-based voluntary counselling and testing site for MSM indicated slight differences in the association of specific meeting places with the risk of new diagnosis of a bSTI or of HIV. ${ }^{9}$

The expanding opportunities to communicate online make it easier for MSM, particularly those not living in large cities with an array of established gay venues, to find and meet new partners. ${ }^{10}$ A shift from using less effective to more effective means of partner seeking (eg, by using GPS-based smartphone applications for dating casual sex partners) may contribute to increasing numbers of partners and consequently to an increase of new diagnoses of STI and HIV among MSM.

In this analysis, we focus on the impact of meeting locations on the probability of being diagnosed with a bSTI in the previous 12 months.

\section{METHODS}

\section{Survey procedures}

Data for this analysis were collected with an online survey targeting MSM living in Germany; the survey was online from 04/11/2013 until 13/01/2014. For a detailed description of the survey and the survey procedures (see online supplementary file).

\section{Measures}

The main outcome of interest in our analysis is selfreported diagnosis of a bSTI (syphilis, gonorrhoea and chlamydia) within the previous 12 months.

Measures used as independent variables in this analysis are: (1) Place where the last non-steady anal sex partner (within the previous 12 months) was met (for categories, see table 1; for multivariate analyses, response options 'not explicitly gay place' and 'another place' were merged); (2) HIV serostatus disclosure and condom use with the last non-steady anal sex partner. The last sexual encounter with a non-steady sex partner was classified as HIV seroconcordant if the reported HIV serostatus of the partner was the same as the serostatus reported by the respondent, as serodiscordant if the respondents reported a different HIV serostatus than his partner, and as non-concordant for any other combination of known and unknown HIV test results; (3) Self-reported HIV status (dichotomised); (4) Size of city of residence (three categories); (5) Number of sex partners in the previous 12 months (five categories); (6) Age group (four categories).

\section{Statistical analysis}

In bivariate analysis, we first looked-stratified by HIV status-at distribution by venues where the last AI partner was met, taking meeting partners online as the reference group.

Then we looked-by HIV status and place of meeting the last non-steady sex partner-at: Diagnosis of a bSTI ; median number of sex partners in the previous 12 months; age group; size of the place of residence; HIV serostatus communication; and condom use at last AI with a non-steady sex partner.

We constructed two different multivariate logistic regression models with diagnosis of a bSTI in the previous 12 months as the outcome variable:

Model 1 assumes that the distinct distribution patterns of the explanatory variables we looked at are intrinsic characteristics associated with meeting venues; for example, sex venues and social venues for MSM are generally localised in larger cities; sex venues are predominantly frequented by men engaging in sex with multiple partners, and serostatus disclosure is uncommon; meeting partners online or on smartphone apps allows a relatively anonymous discussion of HIV serostatus, serostatus concordance and condom use before having sexual intercourse; private sex parties are often organised on the basis of HIV serostatus concordance of participants.

To focus on the effect of the meeting venue, model 1 consequently included only age group and HIV status as additional variables. We distinguished between respondents diagnosed and not diagnosed with HIV in each venue, because we hypothesised that the impact of HIV 
Table 1 History of bSTI diagnosis, and demographic and behavioural characteristics of survey respondents, by HIV status and place of meeting the last non-steady anal sex partner, German MSM online survey 2013

\begin{tabular}{|c|c|c|c|c|c|c|c|c|c|}
\hline & \multicolumn{8}{|c|}{ Place meeting the last non-steady anal sex partner } & \multirow[b]{2}{*}{ Total } \\
\hline & Online & $\begin{array}{l}\text { Smartphone } \\
\text { app }\end{array}$ & $\begin{array}{l}\text { Gay sex } \\
\text { venue }\end{array}$ & $\begin{array}{l}\text { Social } \\
\text { venue }\end{array}$ & $\begin{array}{l}\text { Cruising } \\
\text { place }\end{array}$ & $\begin{array}{l}\text { Private sex } \\
\text { party }\end{array}$ & $\begin{array}{l}\text { Non-gay } \\
\text { venue }\end{array}$ & $\begin{array}{l}\text { Other } \\
\text { places }\end{array}$ & \\
\hline \multicolumn{10}{|c|}{ Proportion meeting the last non-steady sex partner at the respective location } \\
\hline Not diagnosed with & 4841 & 369 & 866 & 387 & 257 & 124 & 471 & 484 & 7799 \\
\hline HIV & $62 \%$ & $4.7 \%$ & $11 \%$ & $4.9 \%$ & $3.3 \%$ & $1.6 \%$ & $6.0 \%$ & $6.2 \%$ & \\
\hline \multirow[t]{2}{*}{ Diagnosed with HIV } & 548 & 42 & 268 & 38 & 56 & 53 & 25 & 49 & 1079 \\
\hline & $51 \%$ & $3.9 \%$ & $25 \%$ & $3.5 \%$ & $5.2 \%$ & $4.9 \%$ & $2.3 \%$ & $4.5 \%$ & \\
\hline \multicolumn{10}{|c|}{ Proportion diagnosed with HIV compared with reference group online } \\
\hline & ref & NS & ** & NS & ** & ** & $\circ$ & NS & \\
\hline \multicolumn{10}{|c|}{ Proportion diagnosed with a bacterial STI in recent 12 months } \\
\hline $\begin{array}{l}\text { Not diagnosed with } \\
\text { HIV }\end{array}$ & $5 \%$ & $7.5 \%$ & $7.4 \%$ & $7.2 \%$ & $4.6 \%$ & $3.1 \%$ & $3.8 \%$ & $3.9 \%$ & $327(4.2 \%)$ \\
\hline Diagnosed with HIV & $* \star 20.3 \%$ & **23.3\% & $\star \star 27.2 \%$ & ${ }^{*} 18.4 \%$ & ${ }^{*} 10.5 \%$ & ${ }^{* *} 26.4 \%$ & $(0 \%)$ & $6.1 \%$ & $\begin{array}{l}* * 225 \\
(20.9 \%)\end{array}$ \\
\hline \multicolumn{10}{|c|}{ Median partner number category (previous 12 months) } \\
\hline $\begin{array}{l}\text { Not diagnosed with } \\
\text { HIV }\end{array}$ & $4-5$ & $6-7$ & $8-10$ & $4-5$ & $8-10$ & $8-10$ & $4-5$ & $4-5$ & \\
\hline Diagnosed with HIV & $8-10$ & $11-20$ & $21-30$ & $6-7$ & $11-20$ & $11-20$ & $6-7$ & $8-10$ & \\
\hline \multicolumn{10}{|l|}{ Median age } \\
\hline $\begin{array}{l}\text { Not diagnosed with } \\
\text { HIV }\end{array}$ & 36 & 31 & 44 & 32 & 45 & 43 & 29 & 36 & 36 \\
\hline Diagnosed with HIV & 44 & 39 & 46 & 43.5 & 44 & 45 & 42 & 44 & 44 \\
\hline \multicolumn{10}{|c|}{ Proportion living in a place with less than 100000 inhabitants } \\
\hline $\begin{array}{l}\text { Not diagnosed with } \\
\text { HIV }\end{array}$ & $48.8 \%$ & $40.7 \%$ & $41.5 \%$ & $34.1 \%$ & $54.1 \%$ & $52.4 \%$ & $47.6 \%$ & $51.4 \%$ & $47.2 \%$ \\
\hline Diagnosed with HIV & ${ }^{\circ} 33.2 \%$ & ${ }^{\circ} 21.4 \%$ & ${ }^{\circ} 23.5 \%$ & ${ }^{\circ} 10.5 \%$ & $\left({ }^{\circ}\right) 41.1 \%$ & ${ }^{\circ} 34 \%$ & ${ }^{\circ} 16 \%$ & ${ }^{\circ} 22.4 \%$ & ${ }^{\circ} 29.1 \%$ \\
\hline \multicolumn{10}{|c|}{ Proportion reporting HIV seroconcordance with last non-steady anal sex partner† } \\
\hline $\begin{array}{l}\text { Not diagnosed with } \\
\text { HIV }\end{array}$ & $32.6 \%$ & $29.4 \%$ & $13.4 \%$ & $30.6 \%$ & $19.4 \%$ & $34.1 \%$ & $36.4 \%$ & $37.3 \%$ & $30.3 \%$ \\
\hline Diagnosed with HIV & $\star * 38.2 \%$ & *37.2\% & $\star * 21 \%$ & $28.9 \%$ & $17.5 \%$ & *54.7\% & *36\% & $25 \%$ & **32.6\% \\
\hline \multicolumn{10}{|c|}{ Proportion reporting not having used a condom for anal intercourse $†$} \\
\hline $\begin{array}{l}\text { Not diagnosed with } \\
\text { HIV }\end{array}$ & $29.6 \%$ & $24.2 \%$ & $28.4 \%$ & $26.6 \%$ & $33.9 \%$ & $39.2 \%$ & $30.8 \%$ & $35.4 \%$ & $29.8 \%$ \\
\hline Diagnosed with HIV & ${ }^{\star *} 63.5 \%$ & *51.2\% & **73.7\% & $48.6 \%$ & $64.3 \%$ & ${ }^{\star *} 86.5 \%$ & *54.2\% & * $45.7 \%$ & ${ }^{* *} 65.2 \%$ \\
\hline
\end{tabular}

${ }^{* *}=$ proportion significantly higher; ${ }^{\circ}=$ significantly lower $\left(p<0.001\right.$ for all comparisons). ${ }^{*}=$ significantly higher $(p<0.04) ;{ }^{\circ}=\operatorname{significantly~lower~}(p<0.025) .\left({ }^{\circ}\right) p=0.064$.

tInformation on HIV serostatus communication and condom use with the last non-steady anal sex partner was based on the following series of questions: What did you tell your partner about your own HIV test result? What did you know or think about the HIV test result of your partner? How did you know or why did you think that? Did you have anal intercourse? (specifying whether anal intercourse was receptive or insertive). Did he use a condom? Did you use a condom?

bSTI, bacterial sexually transmitted infections; MSM, men having sex with men; NS, not significant. 
status would be different by meeting place. The reference category of model 1 are HIV-undiagnosed MSM aged 20-29 years who met their last non-steady anal sex partner online.

Model 2 included additional variables (number of partners in the previous 12 months (reference: 2-5); HIV concordance at last AI (reference: HIV status unknown); condom use at last AI; city size (reference: $100000-500000$ )).

\section{RESULTS}

The online questionnaire was completed by 16734 MSM living in Germany. A previous diagnosis of HIV was reported by 1427 respondents, a previous negative HIV test result by 9886 respondents, and 5341 respondents did not report a previous HIV test. Differences between untested men and men who tested negative for HIV compared with men with an HIV diagnosis were minor in most behavioural parameters analysed, with untested men usually reporting less risky behaviours than men who tested negative. Therefore, we dichotomised HIV status into 'Diagnosed with HIV' and 'Not diagnosed with HIV' for this analysis.

The questions on diagnosis of a bSTI in the previous 12 months and the last AI event were answered by 7799 respondents who were not diagnosed with HIV and 1079 respondents diagnosed with HIV. These 8878 respondents form the final study sample for our analysis.

In our online sample, meeting the last non-steady anal sex partner online was the most frequent mode of meeting non-steady partners, followed by gay sex venues. Other venues were each reported by $2-6 \%$ of the respondents. Sex-focused venues such as sex venues, cruising places and private gay sex parties were mentioned more frequently by respondents diagnosed with HIV (see table 1).

\section{Differences by HIV status}

The proportion reporting diagnosis of a bSTI in the recent 12 months was twofold to fourfold higher among men diagnosed with HIV, except for non-gay venues, for which the number of HIV-diagnosed men meeting their last non-steady partner was small (see table 1).

The partner number categories reported by respondents diagnosed with HIV were consistently one to two categories higher. HIV serostatus communication was reported slightly more often by respondents diagnosed with HIV across all types of venues, with characteristic patterns in different types of venues.

Respondents diagnosed with HIV were older than respondents not diagnosed with HIV, independent of venue. Respondents using smartphone apps had the lowest median age independent of HIV serostatus. Participants with an HIV diagnosis less often lived in cities with less than 100000 inhabitants.

\section{Differences by meeting venue}

Serostatus was relatively frequently communicated at private sex parties, in non-gay settings and online. It was relatively rarely communicated in gay sex venues and at cruising sites.

Condom use was in general much lower for respondents diagnosed with HIV compared with those not diagnosed with HIV, and for both it was lowest at private sex parties. Otherwise, the condom use pattern was different for respondents diagnosed and not diagnosed with HIV: at venues with a low perceived personal responsibility to disclose HIV status (sex venues, cruising sites), low condom use was reported by respondents diagnosed, whereas high condom use was reported by respondents not diagnosed with HIV. For respondents not diagnosed with HIV, meeting venues less associated with the gay subculture (non-gay venues, other places) were associated with relatively low condom use. Notable was the low level of condom use associated with cruising places (see table 1).

In general, mutual serostatus knowledge was associated with lower condom use, regardless of whether serostatus was concordant or discordant (see online supplemental figure). Also, condom use decreased with increasing knowledge of the non-steady partner (see figure 1).

\section{Results of multivariate analysis}

In model 1, HIV status was the strongest predictor for diagnosis of a bSTI with individuals diagnosed with HIV reporting a greater proportion of bSTIs than individuals without HIV diagnosis. For men not diagnosed with HIV, meeting the last non-steady anal sex partner in a gay sex venue or a gay social venue compared with online was associated with an increased risk for STI diagnosis. Meeting the last partner on a smartphone app was associated with an increased risk (OR 1.48; 95\% CI 0.94 to 2.34), which fell short of statistical significance. Men 45 years and older had a significantly lower risk than men aged 20-29 years.

For men diagnosed with HIV, risk was increased when the last non-steady anal sex partner was met in a gay sex venue, and it was significantly lower when the partner was met at a non-gay or other venue (see table 2).

When we included partner numbers, size of the place of residence and HIV status disclosure in model 2 and tested for the various interactions between the included variables by stepwise inclusion, the effect of venues mostly disappeared, while interactions between HIV status and partner numbers as well as condom use became more important. HIV status remained the strongest predictor for bSTI diagnosis. The effect of age was the same as in model 1. HIV status disclosure was associated with increased odds of bSTI diagnosis, regardless of whether status was concordant or discordant. Increasing partner numbers increased the odds of a bSTI diagnosis, more so for men not diagnosed with HIV than for men diagnosed with HIV. Condom use at last AI had no significant effect on bSTI diagnosis among HIV-undiagnosed men, but for HIV-diagnosed men condoms significantly lowered the risk. With the 
Figure 1 Proportion of survey participants reporting no condom use during last anal intercourse with a non-steady partner stratified by partner knowledge (first time, repeated) and reported HIV seroconcordance*, German MSM online survey 2013.

\section{${ }^{*} H I V$ concordant=respondent} reported the same HIV serostatus as his non-steady partner; HIV seroconcordance unknown=either the respondent has never been tested for HIV or the HIV serostatus of his non-steady partner was not known or HIV serostatus was not disclosed.

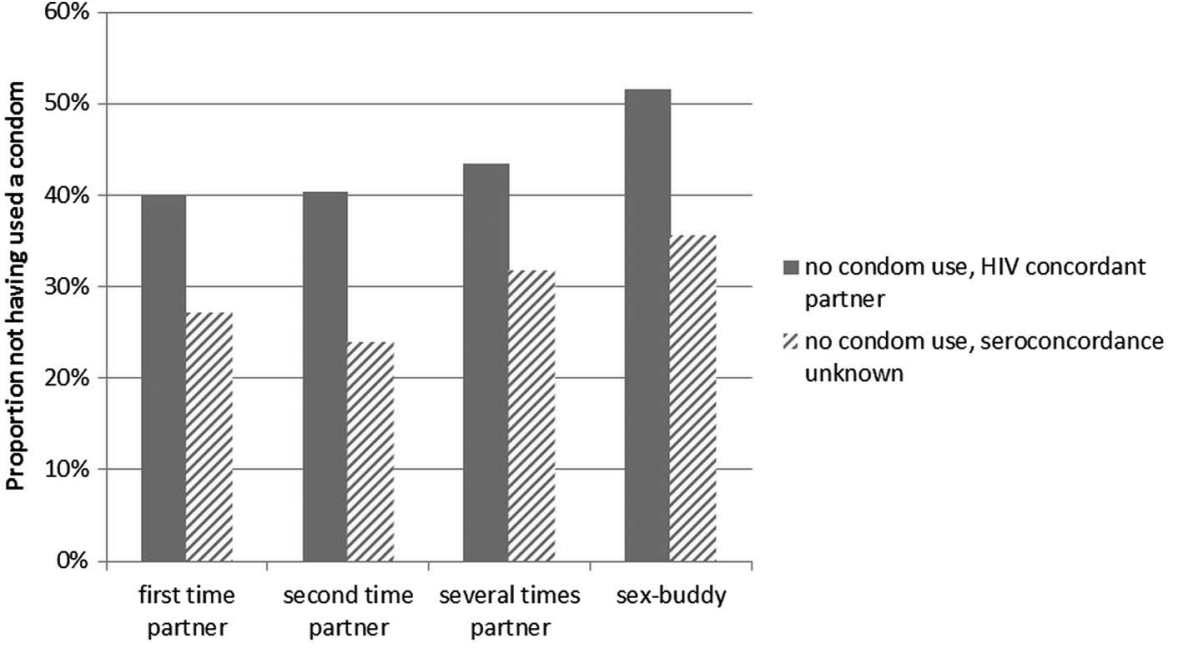

increasing size of the place of residence also, the odds of bSTI diagnosis increased.

Only two meeting venues remained in the model, both associated with a significantly lower risk of bSTI diagnosis: cruising places and non-gay/other venues. Meeting the last non-steady anal sex partner in a gay social or sex venue was still associated with increased odds of having been diagnosed with a bSTI, but this fell short of being statistically significant (see table 3 ).

\section{DISCUSSION}

The type of physical and virtual meeting place with the last non-steady sex partner was strongly associated with the median number of new sex partners in the previous 12 months. This suggests that certain venues facilitate meeting multiple sex partners more than others (eg, sex venues, private sex parties) and/or that such venues are visited preferentially by men interested in having multiple sex partners.

HIV status also has an impact on partner numbers: HIV positive respondents consistently reported higher partner numbers than respondents not diagnosed with HIV, which has also been reported from other studies. ${ }^{11}$ The higher partner numbers of men diagnosed with HIV may have several non-exclusive reasons:

1. Higher partner numbers may be one of the risk factors that contributed to HIV infection.

Table 2 Age-adjusted logistic regression analysis of association of last meeting place with bSTI diagnosis in recent 12 months, German MSM online survey 2013-model 1

\begin{tabular}{|c|c|c|c|c|c|}
\hline Not diagnosed with HIV & OR & $95 \% \mathrm{Cl}$ & Diagnosed with HIV & OR & $95 \% \mathrm{Cl}$ \\
\hline HIV status & & & & 4.93 & 2.80 to 8.66 \\
\hline \multicolumn{6}{|l|}{ Meeting place } \\
\hline Online & refere & & & & \\
\hline Social venue-negative & 1.60 & 1.03 to 2.48 & Social venue-positive & 0.863 & 0.37 to 2.02 \\
\hline Sex venue-negative & 1.88 & 1.37 to 2.57 & Sex venue-positive & 1.52 & 1.07 to 2.14 \\
\hline Private setting-negative & 0.93 & 0.34 to 2.55 & Private setting-positive & 1.46 & 0.76 to 2.79 \\
\hline Cruising place-negative & 1.14 & 0.59 to 2.19 & Cruising place-positive & 0.45 & 0.19 to 1.09 \\
\hline Smartphone app-negative & 1.48 & 0.94 to 2.34 & Smartphone app-positive & 1.07 & 0.51 to 2.25 \\
\hline Other-negative & 0.73 & 0.48 to 1.11 & Other-positive & 0.16 & 0.05 to 0.52 \\
\hline \multicolumn{6}{|l|}{ Age group } \\
\hline $20-29$ & refere & & & & \\
\hline$<20$-negative & 0.62 & 0.35 to 1.09 & $<20$-positive & 1.18 & 0.12 to 11.81 \\
\hline 30-44-negative & 1.07 & 0.82 to 1.39 & 30-44-positive & 1.55 & 0.90 to 2.68 \\
\hline >44-negative & 0.50 & 0.36 to 0.70 & >44-positive & 0.82 & 0.47 to 1.44 \\
\hline \multicolumn{6}{|c|}{$\begin{array}{l}\text { Bold=statistically significant associations }(p<0.05) \text {. } \\
\text { Example of how to read the table: The odds of an MSM diagnosed with HIV who met his last non-steady anal sex partner online having } \\
\text { received a bSTI diagnosis in the recent } 12 \text { months are } 4.93 \text { compared with those of an MSM not diagnosed with HIV. The odds of a man not } \\
\text { diagnosed with HIV who met his non-steady anal sex partner in a gay sex venue were } 1.88 \text { compared with those of a man meeting his last } \\
\text { non-steady anal sex partner online. The odds of a man diagnosed with HIV who met his last non-steady anal sex partner in a gay sex venue } \\
\text { were } 1.52 \text { compared with those of a man diagnosed with HIV and meeting his last partner online, and } 1.52^{*} 4.93=7.49 \text { compared with those of } \\
\text { a man not diagnosed with HIV meeting his last partner online. } \\
\text { bSTI, bacterial sexually transmitted infections; MSM, men having sex with men. }\end{array}$} \\
\hline
\end{tabular}


Table 3 Logistic regression analysis of association of last meeting place with bSTI diagnosis in recent 12 months, German MSM online survey 2013-model 2 (condom use and partner numbers controlled for HIV status (-negative/-positive))

\begin{tabular}{|c|c|c|c|c|c|}
\hline & OR & $95 \% \mathrm{Cl}$ & & OR & $95 \% \mathrm{Cl}$ \\
\hline \multicolumn{6}{|l|}{ HIV status } \\
\hline Positive & & & & 7.02 & 4.13 to 11.93 \\
\hline \multicolumn{6}{|l|}{ Meeting place } \\
\hline Online & reference & & & & \\
\hline Social venue & 1.36 & 0.91 to 2.05 & & & \\
\hline Sex venue & 1.18 & 0.92 to 1.53 & & & \\
\hline Private setting & 0.92 & 0.53 to 1.58 & & & \\
\hline Cruising place & 0.55 & 0.31 to 0.98 & & & \\
\hline Smartphone application & 1.15 & 0.76 to 1.74 & & & \\
\hline Other & 0.64 & 0.43 to 0.97 & & & \\
\hline \multicolumn{6}{|l|}{ Age group } \\
\hline $20-29$ & reference & & & & \\
\hline$<20$ & 0.84 & 0.48 to 1.49 & & & \\
\hline $30-44$ & 1.04 & 0.81 to 1.33 & & & \\
\hline$>44$ & 0.55 & 0.42 to 0.73 & & & \\
\hline \multicolumn{6}{|l|}{ City size } \\
\hline $100000-500000$ & reference & & & & \\
\hline$<100000$ & 0.84 & 0.64 to 1.12 & & & \\
\hline 500 000-1 Million & 1.48 & 1.07 to 2.04 & & & \\
\hline >1 Million & 1.42 & 1.08 to 1.86 & & & \\
\hline \multicolumn{6}{|l|}{ Partner number } \\
\hline 2 to 5 & reference & & & & \\
\hline One-negative & 0.75 & 0.32 to 1.74 & One-positive & 0.47 & 0.06 to 3.71 \\
\hline 6 to 10 -negative & 2.07 & 1.45 to 2.95 & 6 to 10 -positive & 1.46 & 0.85 to 2.50 \\
\hline 11 to 50 -negative & 4.94 & 3.64 to 6.70 & 11 to 50 -positive & 2.00 & 1.24 to 3.24 \\
\hline More than 50 -negative & 7.49 & 4.76 to 11.79 & More than 50-positive & 4.88 & 2.85 to 8.33 \\
\hline \multicolumn{6}{|l|}{ Serostatus communication } \\
\hline Non-concordant & reference & & & & \\
\hline HIV concordant & 1.28 & 1.03 to 1.58 & & & \\
\hline HIV discordant & 2.03 & 1.30 to 3.15 & & & \\
\hline \multicolumn{6}{|l|}{ Condom } \\
\hline Condom use-negative & 0.88 & 0.69 to 1.13 & Condom use-positive & 0.55 & 0.38 to 0.82 \\
\hline
\end{tabular}

2. Restricting partner numbers may be one important strategy to reduce the risk for HIV infection. The diagnosis of HIV removes this necessity.

3. HIV diagnosis may result in disinhibition regarding partner numbers in some and withdrawing from the gay subculture in others. Recruiting survey participants on websites designed primarily to find new partners may introduce a selection bias towards the first group.

Unfortunately, since we have only cross-sectional and no longitudinal data spanning the time of seroconversion and HIV diagnosis, we cannot determine the relative importance of these three reasons.

The reported partner numbers were higher in venues/settings where either serostatus communication/ HIV serosorting was frequent (online and smartphone) or where perceived personal responsibility for serostatus disclosure was low (sex venues). ${ }^{12}$

Cruising places seemed to be the meeting venues which combine the lowest levels of serostatus disclosure and thus probably a relatively high mixing of men diagnosed and not diagnosed with HIV with a relatively low level of condom use. However, risk management in cruising places may operate mainly by avoiding AI in this venue, since only a small proportion of respondents (3\% not diagnosed with HIV, 5\% HIV diagnosed with HIV) met their last non-steady anal sex partner there.

The probability of being diagnosed with a bSTI was much higher among MSM diagnosed with HIV. This is very likely partly explained by higher STI screening frequencies among men diagnosed with HIV and in continuous medical care. ${ }^{13}$ On the other hand, in model 1 , the probability of being diagnosed with a bSTI was higher in MSM visiting sex venues, gay social venues (HIV-negative) and private sex parties (HIV-positive). A higher bSTI risk was associated with higher median partner numbers when meeting the last non-steady sex partner in the respective venue. Serosorting, or preferentially seeking sex partners also infected with HIV to avoid rejection and allow condomless sex without risking HIV transmission, also contributes to a higher risk for STI. ${ }^{14}$ In addition, also known HIV discordance increased bSTI risk in our sample, suggesting selective, HIV-specific precautions. Serostatus disclosure was much more frequent 
when meeting partners online, on a smartphone app or at a private sex party (see reported seroconcordance, table 1). Therefore, it is not surprising that the effect of venues largely disappeared when controlling for partner numbers and serostatus disclosure in model 2.

Higher partner numbers and an increased odds of being diagnosed with a bSTI for smartphone app users compared with men finding their partners online (HIV-undiagnosed) in model 1 may be a consequence of more sexually active men switching to the new tool of smartphone apps preferentially, similar to the early years when the internet became available as a new tool for partner seeking. ${ }^{15}{ }^{16}$ Higher partner numbers and a higher prevalence of ever being diagnosed with an STI have also been reported in a recent publication comparing health outcomes of a smaller sample of 110 MSM who use smartphone apps with MSM who meet partners in other ways. ${ }^{17}$ Another aspect possibly playing a role is the preferential use of smartphone apps in areas with a higher population and MSM density, which are also areas with a higher density of sex venues and higher STI prevalence among MSM. The higher proportion of smartphone app users using a condom for last AI compared with men finding their partners online may be explained by more intense and explicit online communication compared with smartphone app communication, making men communicating with their potential partners online more confident in being able to determine HIV infection risks associated with their partners.

New technologies like GPS-based smartphone apps seem to improve opportunities to find new sex partners compared with seeking partners on gay websites (particularly for younger men and men living in densely populated areas).

\section{Limitations}

There are several limitations to consider when interpreting the results of our analysis. A common limitation for almost all studies among MSM is the lack of a representative sampling frame. Our sample is an online convenience sample, and we cannot claim that our findings are representative of the whole MSM population. Self-selection biases common to online surveys among MSM such as higher education levels compared with the general adult male population probably have been accentuated in this survey by a relatively high attrition rate (see online supplemental file 1). An analysis of survey participants who did not complete the survey showed a higher probability of being younger, not gay identified and having lower education levels. In addition, the online survey was not adapted for smartphones; thus, smartphone users were most likely under-represented in the study sample, possibly introducing further self-selection biases.

Another limitation is the reliance on self-reported STI diagnoses. The large differences regarding STI diagnosis rates between men diagnosed and not diagnosed with HIV are partly explained by different access to routine
STI screening: while for men diagnosed with HIV STI testing can be reimbursed as part of regular HIV treatment monitoring, considerable reimbursement barriers for STI screening for men not diagnosed with HIV exist. Owing to the resulting low adequate STI screening frequencies among men without HIV diagnosis, it is most likely that by using self-reported STI diagnosis rates a high proportion of undiagnosed asymptomatic bSTIs among these men is missed. ${ }^{13}$

When analysing the associations between bSTI diagnosis and behaviours during the last episode of AI with a nonsteady partner, we assume these behaviours are representative of the period of STI acquisition on a population level and ignore that STI could also have been transmitted during other occasions and from a steady partner. Finally, recall and social desirability biases have to be expected, since data on diagnoses and behaviours were self-reported.

\section{CONCLUSIONS}

While behaviour patterns associated with STI risk differ according to HIV status and venues visited, this relationship is mediated by factors that contextualise men's encounters (eg, partner numbers, attitudes towards HIV status disclosure, perceptions about condom use and anonymous sex). Although not directly associated with STIs, venues are connected to social-behavioural facets of corresponding sexual encounters and may be important arenas for differential HIV and STI education, treatment and prevention. Consequently, outreach prevention work in gay venues has long been an important component of HIV prevention for MSM. During the past two decades, advances in communication technology have affected networking patterns, thereby influencing the dynamics of sex partnerships. Close and coordinated cooperation between HIV/STI prevention workers and gay website and smartphone app owners to optimise the technical and design-related opportunities for supporting protective and minimising risk-enhancing behaviours of their customers when seeking new partners should be established and further developed.

Both the venue and individual characteristics must be considered when generating and disseminating STI prevention messaging. ${ }^{18}$ Outreach providers should consider these contextualising aspects when planning interventions in physical and virtual venues.

Acknowledgements The authors would like to acknowledge the work of Michael Bochow who designed and conducted several previous German MSM surveys since the late 1980s and who contributed to this survey with his advice and support. The authors also thank all survey participants who contributed their time to fill in the questionnaire.

Contributors The survey was designed and executed by JD and MK with contributions by UM and MG. The paper was conceived and the manuscript was drafted by UM. Statistical analysis was conducted by UM and MadH. All authors contributed to the writing of the second draft. All authors approved the final manuscript.

Funding This work was supported by the German Federal Centre for Health Education (BZgA) (Grant number 25.5.2.4/13) and by the German Ministry of Health (Grant number IIA5-2014-2514AUK340). 
Competing interests None declared.

Ethics approval Ethical review board of the Charité University Clinic in Berlin.

Provenance and peer review Not commissioned; externally peer reviewed.

Data sharing statement No additional data are available.

Open Access This is an Open Access article distributed in accordance with the Creative Commons Attribution Non Commercial (CC BY-NC 4.0) license, which permits others to distribute, remix, adapt, build upon this work noncommercially, and license their derivative works on different terms, provided the original work is properly cited and the use is non-commercial. See: http:// creativecommons.org/licenses/by-nc/4.0/

\section{REFERENCES}

1. Zhao J, Chen L, Cai WD, et al. HIV infection and sexual behaviors among non-commercial men who have sex with men at different venues. Arch Sex Behav 2014;43:801-9.

2. Vial AC, Starks TJ, Parsons JT. Finding and recruiting the highest risk HIV-negative men who have sex with men. AIDS Educ Prev 2014;26:56-67.

3. Voetsch AC, Lansky A, Drake AJ, et al. Comparison of demographic and behavioral characteristics of men who have sex with men by enrollment venue type in the National HIV Behavioral Surveillance System. Sex Transm Dis 2012;39:229-35.

4. Grov C. HIV risk and substance use in men who have sex with men surveyed in bathhouses, bars/clubs, and on Craigslist.org: venue of recruitment matters. AIDS Behav 2012;16:807-17.

5. Grov C, Agyemang L, Ventuneac A, et al. Navigating condom use and HIV status disclosure with partners met online: a qualitative pilot study with gay and bisexual men from Craigslist.org. AIDS Educ Prev 2013;25:72-85.

6. Grov $\mathrm{C}$, Hirshfield S, Remien $\mathrm{RH}$, et al. Exploring the venue's role in risky sexual behavior among gay and bisexual men: an event-level analysis from a national online survey in the U.S. Arch Sex Behav 2013;42:291-302

7. Grov C, Crow T. Attitudes about and HIV risk related to the "most common place" MSM meet their sex partners: comparing men from bathhouses, bars/clubs, and Craigslist.org. AIDS Educ Prev 2012;24:102-16.

8. Rönn M, White PJ, Hughes G, et al. Developing a conceptual framework of seroadaptive behaviors in HIV-diagnosed men who have sex with men. J Inf Dis 2014;210(Suppl 2):S586-93.

9. Marcus U, Ort J, Grenz M, et al. Risk factors for HIV and STI diagnosis in a community-based HIV/STI testing and counselling site for men having sex with men (MSM) in a large German city in 2011-2012. BMC Inf Dis 2015;15:14

10. Bolding G, Davis M, Hart G, et al. Where young MSM meet their first sexual partner: the role of the Internet. AIDS Behav 2007;11:522-6.

11. Rosenberg ES, Sullivan PS, Dinenno EA, et al. Number of casual male sexual partners and associated factors among men who have sex with men: results from the National HIV Behavioral Surveillance system. BMC Public Health 2011;11:189.

12. O'Leary A, Horvath KJ, Rosser SBR. Associations between partner-venue specific personal responsibility beliefs and transmission risk behavior by HIV-positive men who have sex with men (MSM). AIDS Behav 2013;17:1855-61.

13. Schmidt AJ, Hickson F, Weatherburn $P$, et al. Comparison of the performance of STI Screening Services for gay and bisexual men across 40 European cities: results from the European MSM Internet Survey. Sex Transm Infect 2013;89:575-82.

14. Marcus U, Schmidt AJ, Hamouda O. HIV serosorting among HIV-positive men who have sex with men is associated with increased self-reported incidence of bacterial sexually transmissible infections. Sexual Health 2011;8:184-93.

15. Bolding G, Davis M, Hart G, et al. Gay men who look for sex on the Internet: is there more HIV/STI risk with online partners? AIDS 2005;19:961-8.

16. Downing MJ Jr. Using the internet in pursuit of public sexual encounters: is frequency of use associated with risk behavior among MSM? Am J Mens Health 2012;6:18-27.

17. Lehmiller JJ, loerger M. Social networking smartphone applications and sexual health outcomes among men who have sex with men. PLOS ONE 2014;9:e86603.

18. Kingdon MJ, Storholm ED, Halkitis PN, et al. Targeting HIV prevention messaging to a new generation of gay, bisexual, and other young men who have sex with men. J Health Commun 2013;18:325-42. 